\title{
Ocular manifestations in patients with cerebrovascular accidents
}

\author{
Surajkumar Shobhalal Kuril ${ }^{1}$, Tapan Jakkal $^{2 *}$, Varsha Nandedkar ${ }^{3}$, Mahesh Gutte ${ }^{4}$
}

\author{
1,2Assistant Professor, ${ }^{3}$ Professor and HOD, ${ }^{4}$ Resident Doctor, Department of Ophthalmology, Government Medical College, Aurangabad, \\ Maharashtra, INDIA. \\ Email: tapan jakkal@rediffmail.com
}

\begin{abstract}
Background: Ocular manifestations in patients with cerebrovascular accidents. Aims and objectives: To study the different ocular manifestations in patients with cerebrovascular accidents. Methods and Material: The present case study has included 50 patients who were seen in the outpatient department of ophthalmology of general hospital who have diagnosed cerebrovascular accidents, patients with diagnosed cerebrovascular accidents referred to the department of ophthalmology from department of general medicine. The duration of the study was for 2 years and 50 patients with diagnosed cerebrovascular accidents were enrolled into the study Results: In this study, 47 patients (94\%) had arterial stroke and 3(6\%) had venous stroke. maximum no. of patients were in an age group of 51-60 years in this study. Of the 50 patients in the study, 32(64\%) were males and 18(36\%) were females. This shows higher prevalence of stroke in males. 47 patients $(94 \%)$ had arterial stroke and 3 patients $(6 \%)$ had venous stroke. Thus, majority of patients had arterial stroke. Out of the 47 patients with arterial stroke, $37(78.72 \%)$ had ischemic arterial stroke and 10 patients $(21.27 \%)$ had haemorrhagic arterial stroke. Of the 10 haemorrhagic arterial strokes, majority of the patients had middle cerebral artery territory involvement $(60 \%)$. All patients with middle cerebral artery haemorrhagic stroke had gaze preference to ipsilateral side. Ninety percent patients of haemorrhagic arterial stroke had hypertensive retinopathy of grade II and above. Also in ischemic arterial stroke, most commonly involved territory is that of middle cerebral artery (62.16\%). 39.13\% of patients with middle cerebral artery ischemic stroke had eyes deviated to side of lesion and $60.86 \%$ patients had contralateral homonymous hemianopia. Conclusions: To conclude Retinal vasculature and cerebral vasculature is identical anatomically and embryologically. So regular fundus examinations needed in cases with cerebro vascular accidents to avoid severe damage. Key Words: arterial stroke venous stroke, cerebrovascular accidents
\end{abstract}

*Address for Correspondence:

Dr Tapan Jakkal, Assistant Professor, Department of Ophthalmology, Government Medical College, Aurangabad, Maharashtra, INDIA.

Email: tapan jakkal@,rediffmail.com.

Received Date: 29/03/2019 Revised Date: 13/04/2019 Accepted Date: 30/05/2019

DOI: https://doi.org/10.26611/10091032

\begin{tabular}{|l|l|}
\hline \multicolumn{2}{|c|}{ Access this article online } \\
\hline Quick Response Code: & Website: \\
& www.medpulse.in \\
& \\
\hline
\end{tabular}

\section{INTRODUCTION}

A cerebrovascular accident known as stroke is the rapidly developing loss of brain functions due to disturbance in the blood supply to the brain. Cerebrovascular accident or cerebrovascular insult $(\mathrm{CVI})^{1}$ is also defined as an abnormal condition of the brain characterized by occlusion by an embolus, thrombus, or cerebrovascular haemorrhage or vasospasm, resulting in ischemia of the brain tissues normally perfused by the damaged vessels. The incidence of stroke increases exponentially from 30 years of age, and etiology varies by age ${ }^{2}$ Men are $25 \%$ more likely to suffer strokes than women yet $60 \%$ of deaths from stroke occur in women. Since women live longer, they are older on average when they have their strokes and thus more often killed (NIMH 2002) ${ }^{3}$ The most important modifiable risk factors for stroke are high blood pressure and atrial fibrillation. ${ }^{4}$ Other modifiable risk factors include high blood cholesterol levels, diabetes, cigarette smoking. ${ }^{5}$ (active and passive), heavy alcohol consumption ${ }^{6}$ and drug use, lack of physical activity, obesity and unhealthy diet. ${ }^{7}$ A stroke or cerebrovascular accident (CVA) occurs when the blood supply to a part of the brain is suddenly interrupted by occlusion (an ischemic stroke) ${ }^{1}$ or by 
haemorrhage (a haemorrhagic stroke). The former, ischemia, is a reduction of blood flow due to occlusion (an obstruction). The latter, haemorrhagic stroke, occurs when a blood vessel in the brain bursts, spilling blood into the spaces surrounding the brain cells or when a cerebral aneurysm ruptures. Stroke is literally a "brain attack", caused when the vital supply of blood and oxygen is cut off to part of the brain. If a portion of the brain loses its supply of nutrient-rich blood and oxygen, the bodily functions controlled by that part of the brain (vision, speech, walking, etc.) are impaired. Retinal blood vessels and cerebral small vessels possess similar characteristics anatomically, physiologically and embryologically ${ }^{2}$. Indirectly, retinal microvascular changes may indicate the status of the cerebral vasculature. Retinal microvascular abnormalities reflect cumulative small vessel damage from elevated blood pressure and may reflect subclinical cerebral microvascular changes. Thus, analysis of fundus findings is useful for predicting an atherothrombotic ischemic stroke and planning follow-up examinations. Many cerebrovascular accidents have associated ophthalmologic findings, which are important to recognize for both neurologists and ophthalmologists.

In addition, the ocular manifestations in cerebrovascular accidents can be of great help in finding out the site of lesion. Stroke is a common condition, frequently with significant effects on a patient's ability to live an active and independent life. Anything that may potentially have a beneficial effect on the rehabilitation of such patients should therefore be explored, and as ocular and visual problems are common in patients with stroke, it is important that their implications are understood. This Study endeavours to highlight some important ophthalmologic manifestations associated with various cerebrovascular accidents.

\section{MATERIALS AND METHODS}

Present case study was conducted in a general hospital. Study type- Observational study.

Study duration- January 2017 to December 2018.

Ethical committee permission taken for current study. The present case study has included 50 patients who were seen in the outpatient department of ophthalmology of general hospital who have diagnosed cerebrovascular accidents, patients with diagnosed cerebrovascular accidents referred to the department of ophthalmology from department of general medicine. Patients excluded from the study are patients not willing to be a part of the study, patients with history of trauma to eyes and head injury, patients with presence of other ocular diseases, which confound the findings of cerebrovascular accidents, and patients with infective CNS conditions such as tuberculosis, pyogenic meningitis, etc. The duration of the study was for 2 years (October 2017 to October 2019) and 50 patients with diagnosed cerebrovascular accidents who fulfilled the above-mentioned inclusion and exclusion criteria were enrolled into the study. Consent of the patient is taken in vernacular language understood by the patient and the purpose of the study explained to every patient. In cases of patients who are unconscious, consent of the relatives is taken. Name, age, sex and address of the patient were recorded.

On history, presenting complaints of the patient are noted. Ophthalmic complaints-its onset, duration and progress are noted.

Onset of any visual disturbance if present is noted-whether acute, subacute or chronic.

Other associated symptoms like diplopia, field defect, and altered colour perception are enquired about.

Neurological complaints of the patient (headache, vomiting, weakness, imbalance etc.) their onset, duration and progress are noted. History about various risk factors related to stroke like smoking is enquired about. History regarding onset and duration of diabetes mellitus or hypertension if present is noted. In addition, details are noted whether the patient was under treatment or not for the systemic illness. On examination,

Visual acuity: In cases of patients admitted in the medicine ward, visual acuity was noted bedside in terms of finger counting in meters. If patient is unconscious, vision has not been assessed. In cases of patients who were assessed in ophthalmology department, visual acuity is recorded with the help of Snellen's chart at a distance of 6 meters.

External examination of adnexa is done. Anterior segment evaluation consists of

- For bedridden patients in medicine ward, torchlight examination is done.

- For patients evaluated in ophthalmology out patient department, slit-lamp examination is done.

Pupils: special emphasis is made on examination of pupils.

- Direct and consensual light reflex is checked.

- Relative afferent pupillary defect is noted if present.

Extraocular movements are assessed if patient is conscious and cooperative for the same. Presence or absence of nystagmus is noted. If present, the type of nystagmus- whether jerk or pendular type is noted. Posterior segment of the eye is evaluated with the eyes dilated with a mydriatic agent:

- Direct and indirect ophthalmoscopy is done.

- 90D slit lamp biomicroscopy examination for the evaluation of optic disc and macula is done in cases of patients assessed in outpatient department of ophthalmology. 
- Detailed retinal blood vessel examination is done.

- Hypertensive retinopathy is graded according to Keith Wagner classification

\section{- Grade I}

Thickening, irregularity and tortuosity of retinal arterioles, increased refractiveness

- Grade II

Grade I plus arterio-venous nipping

- Grade III

Grade II plus flame shaped haemorrhage and soft cotton wool exudates.

- Grade IV

Grade III plus papilloedema

Classification of Diabetic Retinopathy is done by:

Early Treatment Diabetic Retinopathy Study (ETDRS)

Levels of Diabetic Retinopathy

Non-proliferative Diabetic Retinopathy (NPDR)

$$
\text { A. Mild NPDR }
$$

- At least one micro aneurysm

\section{B. Moderate NPDR}

- Haemorrhages or micro aneurysms.

- Soft exudates, Venous beading, and intraretinal microvascular abnormalities (IRMAs) definitely present.

\section{Severe NPDR}

- Haemorrhages or micro aneurysms in all four quadrants

- Venous beading in two or more quadrants

- IRMA in at least one quadrant

$$
\text { D. Very Severe NPDR }
$$

- Any two or more of C

Proliferative Diabetic Retinopathy

\section{E. Early PDR}

- New vessels on the retina.

F. High-Risk PDR

- New vessels on the disc (NVD) of $1 / 4$ to $1 / 3$ or more of the disc area or

- Any New vessels and vitreous or pre-retinal haemorrhage

Clinically Significant Macular Edema(any ONE of the following)

- Thickening of the retina located $500 \mu \mathrm{m}$ or less from the center of the macula

- Hard exudates at $500 \mu \mathrm{m}$ or less from the center of the macula with thickening of the adjacent retina

- A zone of retinal thickening, one disc area or larger in size, any portion of which is one disc diameter or less from the center of the macula

In addition to anterior and posterior segment examination, complete neuro- ophthalmic evaluation is done. Visual field testing either is done by confrontation test if patient is unable to come to the outpatient department of ophthalmology or by 30-2 SITA FAST programme on Humphrey automated perimeter machine if the patient is assessed in the outpatient department. If patient is uncooperative then visual fields have not been assessed. Colour vision testing is done by Ishihara chart. Detailed neurological evaluation is done to find out associated motor and sensory deficits. Complete cranial nerve examination is done. Radiological evaluation: CT scan /MRI brain reports of the patient are noted to assess the involved area in the cerebrovascular accidents. The information regarding the vessel responsible for the cerebrovascular accident-whether single or multiple is noted from the report. In cases of venous infarcts, MR venography reports are assessed. Also, the type of cerebrovascular accident - whether haemorrhagic or ischemic is assessed by the report.

Laboratory investigations: The results of any laboratory investigation such as lipid profile, homocysteine level etc. which are significant as risk factors of stroke are noted.

\section{RESULTS AND DISCUSSION}

A cerebrovascular accident also known as stroke is the rapidly developing loss of brain function(s) due to disturbance in the blood supply to the brain. This can be due to ischemia (lack of blood flow) caused by blockage (thrombosis, arterial embolism), or a haemorrhage (leakage of blood). ${ }^{8}$ The present study has been conducted on 50 patients with cerebrovascular accident in a general hospital. Ophthalmic manifestations have been studied in these patients. Cerebrovascular accident is also known as stroke and both these terms have been used interchangeably in the discussion. The patients included in this study are:

- Patients admitted in medicine ward with diagnosed cerebrovascular accidents.

- Patients referred from department of medicine to department of ophthalmology with diagnosed cerebrovascular accidents.

- Patients seen in the outpatient dept. of ophthalmology of a general hospital who have diagnosed cerebrovascular accidents

In this study, patients are divided into two groups -arterial and venous stroke. The patients with arterial stroke have been further divided into ischemic and haemorrhagic stroke ${ }^{9}$. Patients with venous stroke usually have both ischemic and haemorrhagic components. In this study, 47 patients (94\%) had arterial stroke and 3(6\%) had venous stroke .This shows that venous infarcts are much less common as compared to arterial infarcts.

\begin{tabular}{ccc}
\multicolumn{3}{c}{ Age epidemiology of stroke. } \\
\hline Age group (years) & Arterial Stroke & Percentage \\
\hline $31-40$ & 1 & $2.12 \%$ \\
$41-50$ & 2 & $4.25 \%$ \\
\hline
\end{tabular}




\begin{tabular}{ccc}
\hline $51-60$ & 27 & $57.44 \%$ \\
$61-70$ & 14 & $29.78 \%$ \\
$71-80$ & 3 & $6.38 \%$ \\
\hline
\end{tabular}

Age wise classification of venous stroke shows that all the three patients $(100 \%)$ of venous stroke belonged to an age group of 31-40 years This shows that patients of venous stroke belong to a younger age group. In 1992, Ameri and Bousser ${ }^{10}$ reported a uniform age distribution in men with cerebral venous thrombosis, while $61 \%$ of women with cerebral venous thrombosis were aged 20-35 years. Age wise classification of arterial stroke showed that out of 47 patients, $1(2.12 \%)$ belonged to an age group of $31-40$ years, 2 (4.25\%) belonged to an age group of 41-50 years, $27(57.44 \%)$ belonged to an age group of 51-60, 14 (29.78\%) belonged to an age group of 61-70 years of age and $3(6.38 \%)$ belonged to an age group of 71-80 years. Thus, maximum no. of patients were in an age group of 5160 years in this study. Age wise classification of ischemic arterial stroke showed that 1 patient belonged to an age group of 41-50 years, 22 patients belonged to an age group of 51-60 years, 13 patients belonged to an age group of 6170 years and patients belonged to an age group of 71-80 years. Age wise classification of haemorrhagic arterial stroke showed 1 patient belonged to an age group of 31-40 years, 1 patient belonged to an age group of 41-50 years, 5 patients belonged to an age group of 51-60 years, 1 patient belonged to an age group of 61-70 years and 2 patients belonged to an age group of 71-80 years.

Thus, maximum no. of patients were in an age group of 5160 years in this study. As per the study conducted by" National Institute of Neurological Disorders and Stroke (NINDS) (1999) ${ }^{9}$ advanced age is one of the most significant stroke risk factors. $95 \%$ of strokes occur in people age 45 and older.

\begin{tabular}{ccc}
\multicolumn{3}{c}{ Table 2: } \\
\hline & Sex epidemiology of stroke \\
\hline Males & 32 & Percentage \\
Females & 18 & $64 \%$ \\
\hline
\end{tabular}

Of the 50 patients in the study, 32(64\%) were males and $18(36 \%)$ were females This shows higher prevalence of stroke in males. According to a study done by Peter Appelros, Birgitta Stegmayr, et al. on sex differences in stroke epidemiology ${ }^{11}$, male stroke incidence rate was $33 \%$ higher and stroke prevalence was $41 \%$ higher than the female, with large variations between age bands and between populations. Similarly a study conducted by National Institute of Neurological Disorders and Stroke (NINDS) (1999) ${ }^{9}$ men are 25\% more likely to suffer strokes than women.

Sex epidemiology of venous stroke

In venous stroke of this study, there were two females $(66.6 \%)$ and one male (33.3\%). According to the results of the International Study on Cerebral Vein and Dural Sinus Thrombosis (ISCVT) ${ }^{12}$ cerebral venous sinus thrombosis is much more common in women than men with a ratio of approximately 3 to one.

Sex epidemiology of arterial stroke

Out of 47 patients with arterial stroke, $31(65.95 \%)$ were males and $16(34.04 \%)$ were females. Thus, higher prevalence of arterial stroke is seen in males.

A. Venous stroke:

Cerebral venous and sinus thrombosis (CVST) is a relativelyrare subtype of stroke.

Table 3: Patient characteristics in venous stroke

$\begin{array}{cc}\text { Patient } & \text { Venous Strokes } \\ \text { Characteristics } & 3 \\ \text { No. of patients } & \leq 40 \mathrm{yrs}\end{array}$

patients

Sex of patients

Risk Factors

Location of stroke
Females: 2 (66.66\%)

Males: 1 (33.33\%)

-Hyperhomocysteinemia

- Post partum

-Oral Contraceptive Pills

Superior Sagittal Sinus Thrombosis $(100 \%)$
There are 3 patients $(6 \%)$ with venous stroke in this study .Usually venous stroke has both ischemic and haemorrhagic stroke. In this study all the three of the patients belonged to an age group of 31-40 year age group, thus denoting predominance in a younger age group. In 1992, Ameri and Bousser reported a uniform age distribution in men with cerebral venous thrombosis, while $61 \%$ of women with CVT were aged $20-35$ years ${ }^{10}$. There were two females $(66.6 \%)$ and one male $(33.3 \%)$ with venous stroke. According to the results of the International Study on Cerebral Vein and Dural Sinus Thrombosis (ISCVT) ${ }^{12}$ cerebral venous sinus thrombosis is much more common in women than men with a ratio of approximately 3 to one. All three patients in this study had superior sagittal sinus thrombosis. According to the study done by Ameri and Bousser ${ }^{10}$, the superior sagittal and lateral sinuses are commonly (70\%) individually involved by thrombosis. Risk factors seen in the study are hyperhomocysteinemia, postpartum state and intake of oral contraceptives. According to a study done by Canu C, Barinagarrementeria $\mathrm{F}$, on cerebral venous thrombosis in 1993 association with pregnancy and puerperium has been found ${ }^{13}$. Oral contraceptives are also associated with higher risk of cerebral venous thrombosis ${ }^{14}$. Also in a study done by Martineli I, Battaglioli T, Pedotti P, Cattaneo M, Mannucci PM, hyperhomocystinemia is a significant risk factor in cerebral venous thrombosis ${ }^{15}$.

Ophthalmic manifestations in venous stroke 
All three patients $(100 \%)$ of superior sagittal sinus thrombosis had visual acuity of 6/6 in both eyes. Papilloedema in Superior Sagittal Sinus Thrombosis All three patients $(100 \%)$ in the study had papilloedema on fundus examination. Enlargement of blind spot was seen on testing of visual fields. Thus, papilloedema is an important clinical manifestation of superior sagittal sinus thrombosis. One patient (33.33\%) had bilateral abduction deficit due to sixth nerve palsy In a prospective study comprising of 61 consecutive patients with confirmed diagnosis of cerebral venous sinus thrombosis in south of Iran done by Hossein Azin and NahidAshjazadeh 16 showed that $62.3 \%$ patients had papilloedema. In the same study cranial nerve involvement was found in $31.1 \%$ of patients.

B. Arterial stroke:

47 patients in the study had arterial stroke. Out of these 47 patients $37(78.72 \%)$ had ischemic stroke and $10(21.27 \%)$ had haemorrhagic stroke. Thus in this study, ischemic arterial stroke is more common than haemorrhagic arterial stroke.

a. Haemorrhagic arterial stroke

Of the 10 haemorrhagic arterial strokes, various arterial systems affected are as follows

The middle cerebral artery territory (MCA) is involved in 6 patients $(60 \%)$,posterior communicating artery territory $(\mathrm{PCOM})$ is involved in $1(10 \%)$ patient, posterior cerebral artery territory (PCA) is involved in 1 patient $(10 \%)$ and vertebro- basilar artery territory is involved in 2 patients $(20 \%)$.

Ocular manifestations seen in haemorrhagic stroke according to the territory of circulation is as follows

1. Middle cerebral artery related haemorrhagic stroke:

Six patients $(60 \%)$ had bleed in the territory supplied by the middle cerebral artery Five patients $(83.33 \%)$ had gangliocapsular bleed. Ocular manifestations consisted of ocular deviation to the same side as that of bleed in all five patients. Two patients also had ipsilateral dilated pupil probably due to brainstem compression effects. According to a study done on patients with putaminal haemorrhage by Hier DB, Davis KR, et al. ${ }^{17}$, the common clinical manifestations seen in these patients include homonymous hemianopia, conjugate deviation of the eyes toward the side of the lesion, in the dominant hemisphere hematomas, or hemi-inattention in non-dominant lesions. .A study done on patients with caudate haemorrhage by Stein RW, Kase CS, Hier DB et al.. ${ }^{18}$ showed similar manifestations like that with putaminal haemorrhage. One patient $(16.66 \%)$ in the study had parieto temporal lobar haemorrhage. Ocular manifestation included gaze preference to the side of lesion. Thus, all patients with middle cerebral artery haemorrhagic stroke had gaze preference to ipsilateral side.

2. Posterior communicating artery related haemorrhagic stroke:

One female patient $(10 \%)$ had right posterior communicating artery aneurysm rupture with subarachnoid haemorrhage Ophthalmic manifestations in this patient included cranial nerve III palsy with ipsilateral ptosis with fixed dilated pupil. All extraocular movements of right eye were restricted except for abduction and intorsion. The patient had bilateral papilloedema on fundus examination. Due to anatomically close relationship between posterior communicating artery and third cranial nerve, third cranial nerve palsy is a common feature. In a study done on aneurysms of the posterior communicating artery and oculomotor paresis by S. R. Soni ${ }^{19}$ there was a ratio of four females to one male. $34 \%$ of patients in his study had an oculomotor paresis.

3. Posterior cerebral artery related
haemorrhagic stroke

One patient $(10 \%)$ had posterior cerebral artery related haemorrhagic stroke. Area involved in this patient is thalamus.

Ocular manifestation present in the patient with right posterolateral thalamic hematoma showed ocular skew deviation with left eye being downward and medially and nonreactive pupils.

A prospective study of 100 patients of thalamic haemorrhage done by Kumral E, Kocaer T, Ertübey NO, Kumral K. showed that all patients with posterolateral thalamic haemorrhage ${ }^{20}$ had severe sensorimotor deficit. Several variants of vertical gaze dysfunction, skew ocular deviation, gaze preference toward the site of the lesion, and miotic pupils were frequent in posterolateral thalamic haemorrhage, particularly in the large type.

4. Vertebrobasilar arterial haemorrhagic stroke:

Two patients with involvement of this vessel had following manifestations:

a. Pontine haemorrhage: One patient in the study had pontine haemorrhage.

Patient was uncooperative for checking of visual acuity and visual fields. Patient had pinpoint pupils reacting to light. Doll's eye movement was absent.

b. Cerebellar haemorrhage:

Patient had ocular bobbing. The patient also had bilateral papilloedema with grade IV hypertensive retinopathy. Hypertension is known to be a risk factor for haemorrhagic stroke. In a study done by E.Peter Bosch, Stephen S.Kennedy, ocular bobbing was found as an ocular manifestation in-patient with acute cerebellar haemorrhage ${ }^{21}$. The study concluded that ocular bobbing 
is not specific for intrapontine destruction but can be seen in cerebellar lesions as well.

Correlation of hypertension and hypertensive retinopathy in patients with haemorrhagic arterial stroke:

Of the ten patients with haemorrhagic arterial stroke, seven patients $(70 \%)$ had previous history of hypertension and two patients $(20 \%)$ were diagnosed as hypertensive on admission. Thus, nine $(90 \%)$ out of ten patients were hypertensive. All nine patients had hypertensive retinopathy. $4(44.4 \%)$ patients had grade II hypertensive retinopathy, $2(22.2 \%)$ patients had grade III hypertensive retinopathy and $3(33.3 \%)$ patients had grade IV hypertensive retinopathy .Thus, ninety percent patients of haemorrhagic arterial stroke had hypertensive retinopathy of grade II and above Importantly, two patients who were diagnosed hypertensive on admission also had hypertensive retinopathy. Thus, an ophthalmologist can aid in diagnosing hypertensive retinopathy in undiagnosed asymptomatic hypertensives. An analysis done by Aoki N 22 had the results as follows: Among the fundamental funduscopic findings, irregular constriction, narrowing and retinal bleeding were high risk factors of cerebral haemorrhage in due order. . Risk of cerebral haemorrhage was significant and highest in grade 3 of Scheie's hypertensive and grade 3 of Keith-Wagener's classifications. The higher the grade of each funduscopic finding and the classifications of the retinal findings, the higher the risk of occurrence of cerebrovascular diseases. Thus, screening of hypertensive retinopathy could help predict stroke.

Correlation of diabetic retinopathy with haemorrhagic arterial stroke:

In this study, only one patient had diabetic retinopathy. The patient also had concurrent hypertensive retinopathy.

a. Ischemic arterial stroke:

37 patients in the study had ischemic arterial stroke .Out of these 37 patients, 23 patients $(62.16 \%)$ patients had middle cerebral artery territory stroke, 10 patients $(27.02 \%)$ had posterior cerebral artery territory stroke, 2 patients $(5.4 \%)$ had both middle cerebral artery and posterior cerebral artery territory stroke and 2 patients (5.4\%) had basilar artery territory stroke.

1. Ophthalmological manifestations in middle cerebral artery related ischemic stroke:

Of the 23 patients affected, 15 patients $(65.21 \%)$ had gangliocapsular infarct, $5(21.73 \%)$ patients had gangliocapsular and frontoparietal infarct, 2 patients $(8.69 \%)$ had parieto temporal infarct and 1 (4.34\%) patient had parieto temporal and frontal infarct Nine patients $(39.13 \%)$ with middle cerebral artery ischemic stroke had eyes deviated to the side of lesion.Fourteen patients
$(60.86 \%)$ had contralateral homonymous hemianopia as a manifestation of stroke.

2. Ophthalmic manifestations of posterior cerebral artery ischemic stroke:

There were ten patients $(27.02 \%)$ with posterior cerebral artery stroke. Of the 10 patients with posterior cerebral artery stroke, 5 patients $(50 \%)$ had pure occipital infarcts, $2(20 \%)$ patients had both occipital + thalamic infarcts, 1 patient $(10 \%)$ had pure thalamic infarct and 2 patients had midbrain infarct $(20 \%)$.

a. Occipital lobe ischemic infarct:

There were seven patients $(70 \%)$ with occipital infarct. Five of these patients (50\%) had only occipital infarct and two $(20 \%)$ had combined thalamic and occipital infarct. All seven patients $(100 \%)$ had contralateral homonymous hemianopia. Five of these patients could have their visual fields tested on automated perimeter. Their visual fields showed contralateral homonymous hemianopia with macular sparing. Five patients had left occipital lobe involvement (dominant hemisphere). The dominance was determined by right-handedness of the patients. Four $(80 \%)$ of these five patients showed altered colour perception In addition, patients with left occipital lobe infarct had alexia (inability to read) According to a study by Mohr JP, Leicester J, Stoddard LT, Sidman M. et al. right hemianopia, memory and colour deficits are known to occur in circumscribed left posterior cerebral artery territory infarction. ${ }^{23}$

\section{b. Thalamic ischemic infarct:}

Three patients of the ten posterior cerebral artery ischemic stroke had thalamic infarct. Two patients $(20 \%)$ had combined occipital and thalamic infarct. One patient $(10 \%)$ had bilateral Para median thalamic infarct. Patients with combined thalamic and occipital infarct had contralateral homonymous hemianopia. The patient with bilateral Para median thalamic infarct had vertical gaze palsy with restricted elevation and depression. The patient also had amnesia. In a study done by Massimo Gentilini, Ennio De Renzi, GirolamoCrisi et al., eight cases of bilateral Para median thalamic infarcts have been studied ${ }^{24}$.Five out of these eight cases had vertical gaze palsy as a manifestation.

c. Midbrain ischemic infarct:

Two patients $(20 \%)$ of the ten posterior cerebral artery ischemic strokes had midbrain infarct. Both patients had right midbrain infarct with ipsilateral third nerve palsy. Ophthalmic manifestations included ipsilateral ptosis, dilated pupil and restriction of all extraocular movements except abduction and intorsion on the same side Both patients had contralateral left sided hemiplegia. One of the patients also had ipsilateral tremor. Thus, midbrain infarcts cause ophthalmic manifestations related to third nerve 
palsy. A clinical and neuroradiologic study done by Yamana T, Murakami N, Itoh E, Takahashi $\mathrm{A}^{25}$ concluded that the five cases of Weber's syndrome had ipsilateral oculomotor nerve palsy and contralateral hemiparesis. The careful clinical observation of the neurological features of affected patients guided to the obvious site of lesion in the midbrain. In addition, the patient with ipsilateral oculomotor nerve palsy with ipsilateral tremor fit into category of Benedict's syndrome.

3. Ophthalmological manifestations of basilar artery ischemic stroke:

Two patients $(5.4 \%)$ in this study had basilar artery related ischemic stroke. Both the patients had pontine infarct The patient with right inferior pons infarct had right abducens nerve palsy with right abduction deficit. The patient also had right facial nerve palsy of the lower motor neuron type with weak orbicularis action. The other patient with right basal tegmental Para median pontine infarction showed right internuclearophthalmoplegia with restriction of adduction of right eye with end gaze nystagmus of left eye on attempted levoversion. A study done by Satoshi Kataoka, MD; Ariyuki Hori, MD; Tomoyasu Shirakawa, MD Genjiro Hirose for neurological topographical correlation on fifteen patients of basal tegmental Para median pontine infarction ${ }^{26}$ showed that patients presented with hemiparesis and horizontal gaze abnormalities,including abducent nerve palsy $(n=1)$, internuclearophthalmoplegia(INO) $(n=5)$, horizontal gaze palsy $(n=1)$, one-and-a-half $\operatorname{syndrome}(n=1)$, and superficial or proprioceptive sensory dysfunction $(n=8)$.

4. Ophthalmological manifestations of mixed middle cerebral artery and posterior cerebral artery infarct:

Two patients $(5.4 \%)$ in this study belonged to this group. One patient had right parieto occipital infarct. The ophthalmic manifestations include contralateral homonymous hemianopia. The patient also had contralateral visual neglect associated with dressing apraxia. The other patient had left parieto occipital infarct with bilateral frontal horns of lateral ventricle involved. The patient on carotid Doppler also showed left carotid artery stenosis. The patient presented with left eye being completely blind (perception to light negative) and in right eye, visual acuity was 6/12. On fundus examination patient had left central retinal artery occlusion. The right eye showed grade II hypertensive retinopathy. The patient also had aphasia. The internal carotid artery is the source of blood supply for both- the eyes and the brain .Insufficiency of carotid artery can lead to both central retinal artery occlusion and stroke. A study was done by Matysik A, Gerkowicz M, Lewandowska-Furmanik $M^{27}$. The goal of this study was the assessment of the most common coexisting systemic diseases in patients with the occlusion of retinal arteries. The most common concomitant diseases were hypertension, cardiovascular diseases and brain stroke. The most frequent reason of death was cerebral stroke. Cardiovascular and cerebrovascular examinations were recommended for patients with retinal emboli.

Correlation of hypertension and hypertensive retinopathy in patients with ischemic arterial stroke In patients with ischemic arterial stroke, out of 37 patients, 30 patients $(81.08 \%)$ were hypertensive. 14(46.66\%) patients had history of hypertension and 16 patients $(53.33 \%)$ were diagnosed hypertensive on admission. Thus, majority of the patients with cerebrovascular accidents had hypertension diagnosed on admission in this study. 19 of these 30 patients $(63.33 \%)$ with hypertension had hypertensive retinopathy, 7 patients $(23.33 \%)$ had both diabetic and hypertensive retinopathy and 4 patients $(13.33 \%)$ had normal fundus findings Of the 16 patients who were diagnosed hypertensive on admission, 13 patients had hypertensive retinopathy and 3 patients had normal fundus. Thus, fundus examination can aid in diagnosing hypertension in asymptomatic hypertensive. Thus, 26 patients of 30 patients $(86.66 \%)$ with hypertension had hypertensive retinopathy. Of these 26 patients, 3 patients (11.5\%) had grade I hypertensive retinopathy, 19 patients $(73.07 \%)$ had grade II hypertensive retinopathy and 4 patients $(25 \%)$ had grade III hypertensive retinopathy. Majority of patients had grade II hypertensive retinopathy. Thus, hypertensive retinopathy is associated with cerebrovascular accidents. A study by Julia Slark ${ }^{28}$ et al. to determine if hypertensive retinopathy (HTR) is an indicator of silent brain infarction (SBI) in asymptomatic hypertensive subjects using MRI and retinal photography revealed that HTR is a predictor of stroke and the higher the grade of HTR, the more prevalent SBI than in persons with normal retina (by linear association test, $\mathrm{p}=0.001$ ). Thus, serial fundus examinations in hypertensive patients are necessary to help predict stroke. An ophthalmologist plays an important role in diagnosing and classifying the severity of hypertensive retinopathy. Appropriate measures can then be taken to control hypertension.

Hypertensive Retinopathy

Correlation of diabetes mellitus and diabetic retinopathy in patients with ischemic arterial stroke Out of the 37 patients with ischemic arterial stroke, 16 patients (43.24\%) had diabetes mellitus. All these patients had previous history of diabetes mellitus.13 $(81.25 \%)$ of these 16 patients were on treatment and $3(18.75 \%)$ were not on treatment Out of 16 patients with diabetes mellitus, 5 patients $(31.25 \%)$ had diabetic retinopathy, 7 (43.75\%) patients had combined diabetic and hypertensive retinopathy and 4 patients $(25 \%)$ had no evidence of diabetic retinopathy. Thus, 12 patients out of 16 patients 
(75\%) who were diabetic had diabetic retinopathy. Out of these 12 patients, 2 patients $(16.66 \%)$ had mild nonproliferative diabetic retinopathy, 9 patients $(75 \%)$ had moderate non-proliferative diabetic retinopathy and 1 patient $(8.33 \%)$ had severe non-proliferative diabetic retinopathy. Thus, majority of patients had moderate nonproliferative diabetic retinopathy. A study has been done to establish the relationship between diabetic retinopathy and ischemic stroke by Cheung $\mathrm{N}$, Rogers $\mathrm{S}^{29}$ et al.. It concluded that diabetic retinopathy predicts incident ischemic stroke in people with diabetes, independent of other risk factors. Thus, screening for diabetic retinopathy can help in taking appropriate measures for control if diabetic retinopathy and can help in reducing the incidence of stroke.

Diabetic Retinopathy

Correlation of other risk factors with cerebrovascular accidents:

4 patients $(8 \%)$ of the 50 patients had smoking as a risk factor.

5 patients $(10 \%)$ of the 50 patients had altered lipid profile.

Thus, awareness among the ophthalmologists as well as the neurologists regarding the association of cerebrovascular accidents with eye manifestations can lead to better patient assessment

\section{CONCLUSION}

Our study was long term about ocular manifestations in cerebrovascular accident patients. Study of various ocular manifestations done with detailed history, total ophthalmic examinations along with radiological as well as neurological examination. Our study highlight the facts that studying the various ophthalmological manifestations in cerebrovascular accidents can help in localizing the level of lesion. Incidence of arterial ischemic stroke is much more than venous stroke in our study. In case of arterial ischemic stroke age group affected between 51-60 yrs. Males are affected more than females. Middle cerebral arterial territory is most commonly affected in arterial ischemic stroke with most common presentation is contralateral homonymous hemianopia with eye deviated to side of lesion. In case of venous stroke most common age group affected is 31-40 yrs. Females are affected more than men. Middle cerebral arterial territory is most commonly involved in venous stroke with papilloedema as most common ocular finding. Most of the patient of hypertension with venous stroke shows hypertensive retinopathy grade 2 and above changes. Diabetes patients show non-proliferative diabetic retinopathy changes. Some patients shows both hypertensive retinopathy and diabetic retinopathy changes. Risk factors associated with venous stroke are hyperhomocysteinemia, oral contraceptive pills and postpartum state found in our study. Other risk factors are altered lipid profile smoking cigarettes also associated with stroke in our study. Retinal vasculature and cerebral vasculature is identical anatomically and embryologically. So regular fundus examinations needed in cases with cerebro vascular accidents to avoid severe damage.

\section{REFERENCES}

1. Mosby's Medical Dictionary, 8th edition. 2009

2. Ellekjær $\mathrm{H}$; Holmen J, Indredavik B, Terent A. "Epidemiology of Stroke in Innherred, Norway, 1994 to 1996: Incidence and 30-Day Case-Fatality Rate". Stroke 28 (11): 2180-2184.

3. National Institute of Neurological Disorders and Stroke (NINDS) "Stroke: Hope Through Research". National Institutes of Health. Available at: http://www.ninds.nih.gov/disorders/stroke/detail_stroke.h tm (last accessed: 6th Dec 2010).

4. "MRC trial of treatment of mild hypertension: principal results. Medical Research Council Working Party". Br. Medical Journal 1985 Jul. 291 (6488): 97-104.

5. Hankey GJ. "Smoking and risk of stroke". Journal of Cardiovascular Risk 1985 July 6 (4): 207-11.

6. Gorelick PB (1987). "Alcohol and stroke". Stroke; a Journal of Cerebral Circulation 18 (1): 268-71.

7. "Cholesterol, diastolic blood pressure, and stroke: 13,000 strokes in 450,000 people in 45 prospective cohorts. Prospective studies collaboration". Lancet 346 (89918992): 1647-53. 1995. [No authors listed]

8. Sims NR, Muyderman H. "Mitochondria, oxidative metabolism and cell death in stroke". Biochimica et Biophysica Acta 1802 (1): 80-91

9. "Brain Basics: Preventing Stroke". National Institute of Neurological Disorders and Stroke. Available at http://www.ninds.nih.gov/disorders/stroke/preventing_str oke.htm (last accessed: 6th Dec 2010)

10. Ameri A, Bousser MG. Cerebral venous sinus thrombosis. Neurol Clin 1992; 10:87-111

11. Peter Appelros; Birgitta Stegmayr, Andreas Terént, Sex Differences in Stroke Epidemiology, Stroke 2009; 40:1082

12. Ferro JM, Canhao P, Stam J, Bousser MG, Barinagarrementeria F; Prognosis of cerebral vein and dural sinus thrombosis: results of the International Study on Cerebral Vein and Dural Sinus Thrombosis (ISCVT). Stroke 2004; 35:664-70

13. Canu C, Barinagarrementeria F. Cerebral venous thrombosis associated with pregnancy and puerperium: a review of 67 cases. Stroke1993; 24:1880-4.

14. de Bruijn S, Stam J, Koopman M, Vandenbrouke J. Case control study of risk of cerebral venous sinus thrombosis in oral contraceptive users who are carriers of hereditary prothrombotic conditions. Br Med J1998; 316:589-92.

15. Martinelli I, Battaglioli T, Pedotti P, Cattaneo M, Mannucci PM. Hyperhomocysteinemia in cerebral vein thrombosis. Blood 2003; 102:1363-6.

16. Azin H, Ashjazadeh N. Cerebral venous sinus thrombosis-clinical features, predisposing and prognostic factors. Acta Neurol Taiwan. 2008 Jun; 17(2):82-7. 
17. Hier DB,Davis KR,Richardson EP Jr,Mohr JP. Hypertensive putaminal haemorrhage.Ann Neurol. 1977 Feb; 1(2):152-9.

18. Stein RW, Kase CS, Hier DB et al.. Caudate haemorrhage. Neurology (NY) 1984; 34:1549.

19. Soni SR. Aneurysms of the posterior communicating artery and oculomotor paresis. Neurol Neurosurg Psychiatry. 1974 Apr; 37(4):475-84.

20. Emre Kumral, Tülay Kocae, Özgen Ertübey, Kamuran Kumral, Thalamic Haemorrhage A Prospective Study of 100 Patients Stroke. 1995; 26:964-970

21. E. Peter Bosch, et al.. Ocular bobbing. The myth of its localizing value .Neurology 1975 Oct; 25(10): 949.

22. Aoki N. Epidemiological evaluation of funduscopic findings in cerebrovascular diseases. I. Funduscopic findings as risk factors for cerebrovascular diseases. Jpn Circ J. 1975 Mar;39(3):257-69.

23. Mohr JP, Leicester J, Stoddard LT, Sidman M. Right hemianopia with memory and colour deficits in circumscribed left posterior cerebral artery territory infarction. Neurology. Nov 1971; 21(11):1104-13

24. Massimo Gentilini, Ennio De Renzi, Girolamo Crisi, Bilateral Para median thalamic artery infarcts: report of eight cases. Journal of Neurology, Neurosurgery, and Psychiatry 1987; 50:900-909.

25. Wade S. Smith et al. cerebrovascular diseases. Harrison's principle of internal medicine 16 edition; $2: 2381-82$.

26. Satoshi Kataoka, Ariyuki Hori, Tomoyasu Shirakawa, Genjiro Hirose, Para median Pontine Infarction Neurological/Topographical Correlation Stroke.aha journals .1997;28:809-815.

27. Matysik A, Gerkowicz M, Lewandowska-Furmanik M. Systemic diseases in patients with central retinal artery occlusion or its branches in the material of Department of Ophthalmology of Medical University of Lublin. Klin Oczna. 2006; 108(7-9):323-6.

28. Julia Slark, Pankaj Sharma. Cerebrovascular Disease and its Relationship to Other Vascular Beds: A Comprehensive Review of the Literature .Vascular Disease Prevention 2009; 6:1-8.

29. Cheung N, Rogers S, Couper DJ, Klein R, Sharrett AR, Wong TY. Is diabetic retinopathy an independent risk factor for ischemic stroke? Stroke. 2007 Feb; 38(2):398401.

\section{Source of Support: None Declared Conflict of Interest: None Declared}

